\title{
Invariants and Infinitesimal Transformations for Contact Sub-Lorentzian Structures on 3-Dimensional Manifolds
}

\author{
Marek GROCHOWSKI ${ }^{\dagger \ddagger}$ and Ben WARHURST ${ }^{\S}$ \\ $\dagger$ Faculty of Mathematics and Natural Sciences, Cardinal Stefan Wyszyński University, \\ ul. Dewajtis 5, 01-815 Waszawa, Poland \\ E-mail:m.grochowski@uksw.edu.pl \\ $¥$ Institute of Mathematics, Polish Academy of Sciences, \\ ul. Sniadeckich 8, 00-950 Warszawa, Poland \\ E-mail:m.grochowski@impan.pl \\ $\S$ Institute of Mathematics, The Faculty of Mathematics, Informatics and Mechanics, \\ University of Warsaw, Banacha 2, 02-097 Warszawa, Poland \\ E-mail: benwarhurst@mimuw.edu.pl
}

Received October 10, 2014, in final form March 30, 2015; Published online April 17, 2015

http://dx.doi.org/10.3842/SIGMA.2015.031

\begin{abstract}
In this article we develop some elementary aspects of a theory of symmetry in sub-Lorentzian geometry. First of all we construct invariants characterizing isometric classes of sub-Lorentzian contact 3 manifolds. Next we characterize vector fields which generate isometric and conformal symmetries in general sub-Lorentzian manifolds. We then focus attention back to the case where the underlying manifold is a contact 3 manifold and more specifically when the manifold is also a Lie group and the structure is left-invariant.
\end{abstract}

Key words: sub-Lorentzian; contact distribution; left-invariant; symmetry

2010 Mathematics Subject Classification: 53B30; 53A55; 34C14

\section{Introduction}

\subsection{Basic notions and motivation}

Sub-Lorentzian geometry is a relatively new subject although it does fall within the scope of broader perspectives on geometry. For instance the work of Berestovskii and Gichev [4] on metrized semigroups is perhaps the broadest perspective one could take on the subject or alternatively a more closely related generalisation is the notion of para-CR-geometry see [18].

The aim in this work is to look specifically at sub-Lorentzian geometry and so in this section we only present the notions that are required for the formulation of the main results of the paper. For more details and facts concerning sub-Lorentzian geometry, the reader is referred to [12] and the references therein (see also [17, 20]). The structures which support the subLorentzian structures in this paper are identical to those which support the analogous structures in the sub-Riemannian setting and so the reader is also referred to the paper of Agrachev and Barilari [1] and the paper of Falbel and Gorodski [8] for background on these structures.

Let $M$ be a smooth manifold. A sub-Lorentzian structure on $M$ is a pair $(H, g)$, where $H$ is a bracket generating distribution of constant rank on $M$, and $g$ is a Lorentzian metric on $H$. A triple $(M, H, g)$, where $(H, g)$ is a sub-Lorentzian structure on $M$, will be called a subLorentzian manifold. 
For any $q \in M$, a vector $v \in H_{q}$ will be called horizontal. A vector field $X$ on $M$ is horizontal if it takes values in $H$. We will denote the set of all local horizontal vector fields by $\Gamma(H)$. To be more precise, $X \in \Gamma(H)$ if and only if $X$ is a horizontal vector field defined on some open subset $U \subset M$.

A nonzero vector $v \in H_{q}$ is said to be timelike (resp. spacelike, null, nonspacelike) if $g(v, v)<0$ (resp. $g(v, v)>0, g(v, v)=0, g(v, v) \leq 0$ ), moreover the zero vector is defined to be spacelike. Similarly, vector fields are categorized analogously according to when their values lie in exactly one of the four categories mentioned above. An absolutely continuous curve $\gamma:[a, b] \longrightarrow M$ is called horizontal if $\dot{\gamma}(t) \in H_{\gamma(t)}$ a.e. on $[a, b]$. A horizontal curve $\gamma:[a, b] \longrightarrow M$ is timelike (spacelike, null, nonspacelike) if $\dot{\gamma}(t)$ is timelike (spacelike, null, nonspacelike) a.e. on $[a, b]$.

If $(H, g)$ is a sub-Lorentzian metric on $M$ then, as shown in [15], $H$ can be represented as a direct sum $H=H^{-} \oplus H^{+}$of subdistributions such that rank $H^{-}=1$ and the restriction of $g$ to $H^{-}$(resp. to $H^{+}$) is negative (resp. positive) definite. This type of decomposition will be called a causal decomposition of $H$. Now, by a time (resp. space) orientation of $(M, H, g)$ we mean an orientation of the vector bundle $H^{-} \longrightarrow M$ (resp. $H^{+} \longrightarrow M$ ). This definition requires some explanations since causal decompositions are not unique. So suppose that we are given two causal decompositions $H=H_{1}^{-} \oplus H_{1}^{+}=H_{2}^{-} \oplus H_{2}^{+}$and that the bundles $H_{i}^{-} \longrightarrow M$ (resp. $\left.H_{i}^{+} \longrightarrow M\right), i=1,2$, are oriented. We say that the two orientations of $H_{1}^{-} \longrightarrow M$ and $H_{2}^{-} \longrightarrow M$ (resp. of $H_{1}^{+} \longrightarrow M$ and $H_{2}^{+} \longrightarrow M$ ) define the same time (resp. space) orientation of $(M, H, g)$ if around any point of $M$ there exist local sections $X_{1}^{(i)}$ of $H_{i}^{-} \longrightarrow M$ which agree with the given orientations of $H_{i}^{-} \longrightarrow M$ (resp. local sections $X_{2}^{(i)}, \ldots, X_{k}^{(i)}$ of $H_{i}^{+} \longrightarrow M$ which agree with the given orientations of $\left.H_{i}^{+} \longrightarrow M\right), i=1,2$, such that $g\left(X_{1}^{(1)}, X_{1}^{(2)}\right)<0$ (resp. $\operatorname{det}\left(g\left(X_{i}^{(1)}, X_{j}^{(2)}\right)_{i, j=2, \ldots, k}>0\right)$; by $k$ we denote the rank of $H$.

Since a line bundle is orientable if and only if it is trivial, time orientability of $(M, H, g)$ is equivalent to the existence of a continuous timelike vector field on $M$. A choice of such a timelike field is called a time orientation of $(M, H, g)$.

Suppose that $(M, H, g)$ is time oriented by a vector field $X$. A nonspacelike $v \in H_{q}$ will be called future (resp. past) directed if $g(v, X(q))<0$ (resp. $g(v, X(q))>0$ ). A horizontal curve $\gamma:[a, b] \longrightarrow M$ is called timelike future (past) directed if $\dot{\gamma}(t)$ is timelike future (past) directed a.e. Similar classifications can be made for other types of curves, e.g. nonspacelike future directed etc. If $q_{0} \in M$ is a point and $U$ is a neighborhood of $q_{0}$, then by the future timelike (nonspacelike, null) reachable set from $q_{0}$ relative to $U$ we mean the set of endpoints of all timelike (nonspacelike, null) future directed curves that start from $q_{0}$ and are contained in $U$.

Now we define a very important notion that will play a crucial role in the sequel. As it is known [12], any sub-Lorentzian structure $(H, g)$ determines the so-called geodesic Hamiltonian which is defined as follows. The existence of the structure $(H, g)$ is equivalent to the existence of the fiber bundle morphism $G: T^{*} M \longrightarrow H$ covering the identity defined by $G(\lambda)=\left(\left.\lambda_{p}\right|_{H_{p}}\right)^{\sharp}$, where $\lambda \in T_{p}^{*} M$ and $\sharp$ denotes the musical isomorphism. In particular, if $v, w$ are any horizontal vectors, then $g(v, w)=\langle\xi, G \eta\rangle=\langle\eta, G \xi\rangle$ whenever $\xi \in G^{-1}(v), \eta \in G^{-1}(w)$. The geodesic Hamiltonian is the map $h: T^{*} M \longrightarrow \mathbb{R}$ defined by

$$
h(\lambda)=\frac{1}{2}\langle\lambda, G \lambda\rangle .
$$

If $X_{1}, \ldots, X_{k}$ is an orthonormal basis for $(H, g)$ with a time orientation $X_{1}$, then

$$
\left.h\right|_{T_{q}^{*} M}(\lambda)=-\frac{1}{2}\left\langle\lambda, X_{1}(q)\right\rangle^{2}+\frac{1}{2} \sum_{i=2}^{k}\left\langle\lambda, X_{i}(q)\right\rangle^{2} .
$$

A horizontal curve $\gamma:[a, b] \longrightarrow M$ is said to be a Hamiltonian geodesic if there exists $\Gamma$ : $[a, b] \longrightarrow T^{*} M$ such that $\dot{\Gamma}=\vec{h}(\Gamma)$ and $\pi(\Gamma(t))=\gamma(t)$ on $[a, b]$; by $\pi: T^{*} M \longrightarrow M$ we denote the canonical projections, and $\vec{h}$ is the Hamiltonian vector field corresponding to $h$. 
Let $\gamma:[a, b] \longrightarrow M$ be a nonspacelike curve. The non-negative number

$$
L(\gamma)=\int_{a}^{b}|g(\dot{\gamma}(t), \dot{\gamma}(t))|^{1 / 2} d t
$$

is called the sub-Lorentzian length of a curve $\gamma$. If $U \subset M$ is an open subset, then the (local) sub-Lorentzian distance relative to $U$ is the function $d[U]: U \times U \longrightarrow[0,+\infty]$ defined as follows: For $q_{1}, q_{2} \in U$, let $\Omega_{q_{1}, q_{2}}^{n s p c}(U)$ denote the set of all nonspacelike future directed curves contained in $U$ which join $q_{1}$ to $q_{2}$, then

$$
d[U]\left(q_{1}, q_{2}\right)= \begin{cases}\sup \left\{L(\gamma): \gamma \in \Omega_{q_{1}, q_{2}}^{n s p c}(U)\right\}: & \Omega_{q_{1}, q_{2}}^{n s p p_{2}}(U) \neq \varnothing, \\ 0: & \Omega_{q_{1}, q_{2}}^{n s p c}(U)=\varnothing\end{cases}
$$

(if $\Omega_{q, q}^{n s p c}(U)$ is non-empty for a $q \in U$ then $d[U](q, q)=\infty$ ). A nonspacelike future directed curve $\gamma:[a, b] \longrightarrow U$ is called a $U$-maximizer if $d[U](\gamma(a), \gamma(b))=L(\gamma)$. It can be proved (see [12]) that every sufficiently small subarc of every nonspacelike future directed Hamiltonian geodesic is a $U$-maximizers for suitably chosen $U$.

Suppose now that we are given two sub-Lorentzian manifolds $\left(M_{i}, H_{i}, g_{i}\right), i=1,2$. A diffeomorphism $\varphi: M_{1} \longrightarrow M_{2}$ is said to be a sub-Lorentzian isometry, if $d \varphi\left(H_{1}\right) \subset H_{2}$ and for each $q \in M_{1}$, the mapping $d \varphi_{q}:\left(H_{1}\right)_{q} \longrightarrow\left(H_{2}\right)_{\varphi(q)}$ is a linear isometry, i.e., for every $v_{1}, v_{2} \in\left(H_{1}\right)_{q}$ it follows that

$$
g_{1}\left(v_{1}, v_{2}\right)=g_{2}\left(d \varphi_{q}\left(v_{1}\right), d \varphi_{q}\left(v_{2}\right)\right) .
$$

Of course, any isometry maps timelike curves from $M_{1}$ to timelike curves on $M_{2}$. The same for spacelike and null curves. Moreover isometries preserve the sub-Lorentzian length of nonspacelike curves. If $\left(M_{i}, H_{i}, g_{i}\right), i=1,2$, are both time- and space-oriented, then we can distinguish among all isometries those that preserve one of the orientations or both of them. More precisely, suppose that $\varphi: M_{1} \longrightarrow M_{2}$ is an isometry. Let $H_{1}=H_{1}^{-} \oplus H_{1}^{+}$be a causal decomposition with given orientation on $H_{1}^{ \pm}$. Let $\left(H_{2}^{-}\right)_{\varphi(q)}=d \varphi_{q}\left(H_{1}^{-}\right)_{q}$, and $\left(H_{2}^{+}\right)_{\varphi(q)}=d \varphi_{q}\left(H_{1}^{+}\right)_{q}, q \in M$. Then $H_{2}=$ $H_{2}^{-} \oplus H_{2}^{+}$is again a causal decomposition where the summands $H_{2}^{ \pm}$inherit the orientation carried from $H_{1}^{ \pm}$by $\varphi$. Now we say that $\varphi$ preserves time (resp. space) orientation if the orientations of $H_{2}^{-}$(resp. $H_{2}^{+}$) induced by $\varphi$ agrees with the time (resp. space) orientation of $\left(M_{2}, H_{2}, g_{2}\right)$. An isometry that preserves time and space orientation will be called a ts-isometry. It is clear that any $t s$-isometry preserves Hamiltonian geodesics, maximizers, and local sub-Lorentzian distance functions. Notice furthermore that the set of all isometries $(M, H, g) \longrightarrow(M, H, g)$ is a Lie group and the set of all $t s$-isometries forms a connected component containing the identity.

A sub-Lorentzian manifold $(M, H, g)$ is called a contact sub-Lorentzian manifold, if $H$ is a contact distribution on $M$. Among sub-Lorentzian manifolds, those which are contact seem to be the easiest to study and hence well known. Contact sub-Lorentzian manifolds are studied for instance in papers $[9,10,11,14,17,19,20,21]$. The investigations go in two directions. The first addresses global aspects, e.g., in [10,11] the Heisenberg sub-Lorentzian metric is treated. More precisely, the future timelike, nonspacelike and null reachable sets from a point are computed, and a certain estimate on the distance function is given. Moreover, it is shown that the future timelike conjugate locus of the origin is zero, while the future null conjugate locus equals the union of the two null future directed Hamiltonian geodesics starting from the origin. In turn, in [20] and [11] it is proved that the set reachable from the origin by future directed timelike Hamiltonian geodesics coincides with the future timelike reachable set from the origin. In [20] the authors also study the set reachable by spacelike Hamiltonian geodesics and prove the uniqueness of geodesics in the Heisenberg case. Next, in the papers [20, 21] the so-called $\mathbb{H}$-type groups (i.e. higher-dimensional analogues of the 3D Heisenberg group) with 
suitable sub-Lorentzian metrics are studied, and the main emphasis is put on the problem of connectivity by geodesics, i.e. given two points $q_{1}, q_{2}$, figure out how many geodesics joining $q_{1}$ to $q_{2}$ exist. A similar problem is also dealt with in [19]. On the other hand, in [17] the group $\mathrm{SL}(2, \mathbb{R})$ with the sub-Lorentzian metric is studied. As it will become clear below, the cases of the Heisenberg group and that of $\operatorname{SL}(2, \mathbb{R})$ are especially interesting for us because these are exactly the cases that arise when the invariant $\tilde{h}$ (defined below) vanishes. The other direction of studies concerns the local situation and is based on the construction (see, e.g., [9, 13, 14]) of local normal forms. Such local normal forms depend on two functional parameters and permit to view general structures as perturbations of flat ones. This fact allows to generalize some global results that hold in the flat case to local results for general structures describable by the mentioned normal forms.

As one can see, problems connected with isometric and conformal symmetry have not been examined in an explicit sense although in broader contexts such as parabolic geometry and Cartan's equivalence, there are applicable results. The aim of this paper is to embark on filling this gap. More precisely, first we construct invariants for contact sub-Lorentzian manifolds $(M, H, g)$ with $\operatorname{dim} M=3$, more or less in the way as it is done in the contact sub-Riemannian case - cf. [1]. Our invariants are: a $(1,1)$-tensor $\tilde{h}$ on $H$ and a smooth function $\kappa$ on $M$. Then, we consider in some detail the case that $M$ is a 3 -dimensional Lie group such that $\tilde{h}=0$. It turns out that in such a case $M$ is locally either the Heisenberg group or the universal cover of $\mathrm{SL}(2, \mathbb{R})$. In these two cases we describe infinitesimal isometries and more generally infinitesimal conformal transformations.

\subsection{The content of the paper}

In Section 2 we construct invariants for $t s$-oriented contact sub-Lorentzian metrics on 3D manifolds. The construction follows the ideas of [1], however the full analogy does not exist due to the special character of indefinite case. Our main invariants for a manifold $(M, H, g)$ are: a smooth $(1,1)$-tensor $\tilde{h}$ on $H$ and a smooth function $\kappa$ on $M$. These invariants provide necessary conditions for two contact sub-Lorentzian manifolds to be locally $t s$-isometric. We also consider another invariant $\chi$ arising from the eigenvalues of $\tilde{h}$ which to a lesser extent also distinguishes the structure. The question as to whether $\{\tilde{h}, \kappa\}$ is a complete set of invariants requires deeper analysis using Cartan's theory and is deferred to a forthcoming paper with Alexandr Medvedev [16].

In Section 3 we define and prove basic properties of infinitesimal sub-Lorentzian isometries and conformal transformations. Then we notice that the invariant $\tilde{h}$ can be expressed in terms of the restricted Lie derivative of the metric $g$ in the direction of the Reeb vector field. The immediate consequence of this latter fact is that the Reeb vector field $X_{0}$ is an infinitesimal isometry if and only if $\tilde{h}$ vanishes identically.

Section 4 covers some other implications of certain combinations of the invariants vanishing. In particular we demonstrate (see Proposition 4.5) that without any assumptions on orientation, the condition $\chi=0$ and $\tilde{h} \neq 0$ implies the existence of line sub-bundle $L \rightarrow M$ of $H$ on which the metric $g$ is equal to zero. We then begin to focus on the condition $\tilde{h}=0$, where $\kappa$ comes to the fore. For example, when $M$ is a simply connected Lie group, we show that $\tilde{h}=0$ and $\kappa=0$ implies that $M$ is the Heisenberg group - cf. Corollary 4.11, and $\tilde{h}=0$ and $\kappa \neq 0$ implies that $M$ is the universal cover of $\mathrm{SL}(2, \mathbb{R})$ - see Corollary 4.12. This contrasts with the sub-Riemannian case where a third group, namely $\mathrm{SU}(2)$, also appears.

Section 6 is devoted to computing infinitesimal isometries and infinitesimal conformal transformations using Cartan's equivalence method and Appendix presents an example of an isometrically rigid sub-Lorentzian structure.

Finally, the appendix presents possible applications of our invariants to a non-contact case. 


\section{Constructing the invariants}

\subsection{Preliminaries}

Let $(M, H, g)$ be a contact sub-Lorentzian manifold, $\operatorname{dim} M=3$, which is supposed to be both time and space oriented or ts-oriented for short. Since $H$ is of rank 2 , any causal decomposition $H=H^{-} \oplus H^{+}$splits $H$ into a direct sum of line bundles. So in this case a space orientation is just a continuous spacelike vector field, and consequently $H$ admits a global basis. Let us fix an orthonormal basis $X_{1}, X_{2}$ for $(H, g)$, i.e.

$$
g\left(X_{1}, X_{1}\right)=-1, \quad g\left(X_{1}, X_{2}\right)=0, \quad g\left(X_{2}, X_{2}\right)=1
$$

where $X_{1}$ (resp. $\left.X_{2}\right)$ is a time (resp. space) orientation. From now on we will work with tsinvariants, i.e., with invariants relative to $t s$-isometries. However, when reading the text the reader will see that the space orientation is only an auxiliary notion here and most of the results do not depend on it (some of them do not depend on an orientation at all).

Let $\omega$ be a contact 1 -form such that $H=\operatorname{ker} \omega$. Without loss of generality we may assume that $\omega$ is normalized so that

$$
d \omega\left(X_{1}, X_{2}\right)=\omega\left(\left[X_{2}, X_{1}\right]\right)=1
$$

Next, denote by $X_{0}$ the so-called Reeb vector field on $M$ which is defined by

$$
\omega\left(X_{0}\right)=1, \quad d \omega\left(X_{0}, \cdot\right)=0 .
$$

It is seen that $X_{0}$ is uniquely determined by $t s$-oriented sub-Lorentzian structure. Using (2.1) it is seen that the action of $\operatorname{ad}_{X_{0}}$ preserves the horizontality of vector fields, i.e.

$$
\operatorname{ad}_{X_{0}}(\Gamma(H)) \subset \Gamma(H) .
$$

Now (similarly as in [1]) we introduce the structure functions. Thanks to (2.2) and (2.1) we have

$$
\begin{aligned}
& {\left[X_{1}, X_{0}\right]=c_{01}^{1} X_{1}+c_{01}^{2} X_{2}, \quad\left[X_{2}, X_{0}\right]=c_{02}^{1} X_{1}+c_{02}^{2} X_{2},} \\
& {\left[X_{2}, X_{1}\right]=c_{12}^{1} X_{1}+c_{12}^{2} X_{2}+X_{0} .}
\end{aligned}
$$

Let $\nu_{0}, \nu_{1}, \nu_{2}$ be the dual basis of 1-forms: $\left\langle\nu_{i}, X_{j}\right\rangle=\delta_{i j}, i, j=0,1,2$. Rewriting (2.3) in terms of $\nu_{i}$ 's we have

$$
\begin{aligned}
& d \nu_{0}=\nu_{1} \wedge \nu_{2}, \quad d \nu_{1}=c_{01}^{1} \nu_{0} \wedge \nu_{1}+c_{02}^{1} \nu_{0} \wedge \nu_{2}+c_{12}^{1} \nu_{1} \wedge \nu_{2}, \\
& d \nu_{2}=c_{01}^{2} \nu_{0} \wedge \nu_{1}+c_{02}^{2} \nu_{0} \wedge \nu_{2}+c_{12}^{2} \nu_{1} \wedge \nu_{2} .
\end{aligned}
$$

Differentiating the first equation in (2.4) we obtain $0=d \nu_{1} \wedge \nu_{2}-\nu_{1} \wedge d \nu_{2}=\left(c_{01}^{1}+c_{02}^{2}\right) \nu_{0} \wedge \nu_{1} \wedge \nu_{2}$ from which it follows that

$$
c_{01}^{1}+c_{02}^{2}=0 .
$$

\subsection{Induced bilinear form and linear operator}

In the introduction we defined the geodesic Hamiltonian $h$ which can be written as

$$
h=-\frac{1}{2} h_{1}^{2}+\frac{1}{2} h_{2}^{2}
$$


where $h_{i}(\lambda)=\left\langle\lambda, X_{i}\right\rangle, i=1,2$. We also consider the function $h_{0}(\lambda)=\left\langle\lambda, X_{0}\right\rangle$ and observe that by definition both $h$ and $h_{0}$ are invariant with respect to $t s$-oriented structure $(H, g)$. Therefore, it is the same with their Poisson bracket $\left\{h, h_{0}\right\}$ which, when evaluated at $q \in M$, gives a symmetric bilinear form

$$
\left\{h, h_{0}\right\}_{q}: T_{q}^{*} M \times T_{q}^{*} M \longrightarrow \mathbb{R} .
$$

If $\lambda \in T_{q}^{*} M$ then $\lambda=\sum_{i=0}^{3} h_{i}(\lambda) \nu_{i}(q)$ and

Lemma 2.1. $\left\{h, h_{0}\right\}_{q}=-c_{01}^{1} h_{1}^{2}+\left(c_{02}^{1}-c_{01}^{2}\right) h_{1} h_{2}+c_{02}^{2} h_{2}^{2}$.

Proof. The formula follows from $\left\{h, h_{0}\right\}=-h_{1}\left\{h_{1}, h_{0}\right\}+h_{2}\left\{h_{2}, h_{0}\right\}$, where we substitute $\left\{h_{i}, h_{0}\right\}(\lambda)=\left\langle\lambda,\left[X_{i}, X_{0}\right]\right\rangle$, and then use $(2.3)$.

In the assertion of Lemma 2.1 and in many other places below we write $c_{j k}^{i}$ for $c_{j k}^{i}(q)$.

It follows that $\left\{h, h_{0}\right\}_{q}(\lambda, \cdot)=0$ whenever $\lambda \in H_{q}^{\perp}$ (by definition $H_{q}^{\perp}$ is the set of such covectors $\lambda \in T_{q}^{*} M$ that $\langle\lambda, v\rangle=0$ for every $v \in H_{q}$ ), so in fact

$$
\left\{h, h_{0}\right\}_{q}: T_{q}^{*} M / H_{q}^{\perp} \times T_{q}^{*} M / H_{q}^{\perp} \longrightarrow \mathbb{R}
$$

Let us recall (mutually inverse) musical isomorphisms determined by the metric $g$; these are $\sharp: H^{*} \longrightarrow H$ and $^{b}: H \longrightarrow H^{*}$, where by definition $\left(\nu_{1}\right)^{\sharp}=-X_{1},\left(\nu_{2}\right)^{\sharp}=X_{2},\left(X_{1}\right)^{b}=-\nu_{1}$, $\left(X_{2}\right)^{b}=\nu_{2}$. Now it is easy to see that the bundle morphism $G: T^{*} M \longrightarrow H$ from the introduction induces for each $q$ a natural identification

$$
F_{q}: T_{q}^{*} M / H_{q}^{\perp} \longrightarrow H_{q}, \quad F([\alpha])=\left(\alpha_{\mid H_{q}}\right)^{\sharp},
$$

where $[\alpha]$ stands for the class of $\alpha \in T_{q}^{*} M$ modulo $H_{q}^{\perp}$; more precisely, $H_{q}^{\perp}$ is spanned by $\nu_{0}$, and $F\left(\left[\nu_{1}\right]\right)=-X_{1}, F\left(\left[\nu_{2}\right]\right)=X_{2}$. This permits us to define a bilinear symmetric form $\bar{h}_{q}$ : $H_{q} \times H_{q} \longrightarrow \mathbb{R}$ by

$$
\bar{h}_{q}(v, w)=\left\{h, h_{0}\right\}_{q}\left(F_{q}^{-1}(v), F_{q}^{-1}(w)\right) .
$$

Its matrix in the basis $X_{1}(q), X_{2}(q)$ is

$$
\left(\begin{array}{cc}
-c_{01}^{1} & -\frac{1}{2}\left(c_{02}^{1}-c_{01}^{2}\right) \\
-\frac{1}{2}\left(c_{02}^{1}-c_{01}^{2}\right) & c_{02}^{2}
\end{array}\right)
$$

Finally we define a linear mapping $\tilde{h}_{q}: H_{q} \longrightarrow H_{q}$ by the following formula:

$$
\tilde{h}_{q}(v)=\left(\bar{h}_{q}(v, \cdot)\right)^{\sharp} .
$$

Using (2.6), it is seen that the matrix of the operator $\tilde{h}_{q}$ in the basis $\left\{X_{1}(q), X_{2}(q)\right\}$ is equal to

$$
\left(\begin{array}{cc}
c_{01}^{1} & \frac{1}{2}\left(c_{02}^{1}-c_{01}^{2}\right) \\
-\frac{1}{2}\left(c_{02}^{1}-c_{01}^{2}\right) & c_{02}^{2}
\end{array}\right) .
$$




\subsection{The $t s$-invariants}

By our construction, the eigenvalues and determinant of $\tilde{h}_{q}$ as well as $\tilde{h}_{q}$ itself, are all invariants

for the $t s$-oriented structure $(H, g)$. Clearly $\operatorname{det} \tilde{h}_{q}=c_{01}^{1} c_{02}^{2}+\frac{1}{4}\left(c_{02}^{1}-c_{01}^{2}\right)^{2}=-\left(c_{01}^{1}\right)^{2}+\frac{1}{4}\left(c_{02}^{1}-\right.$ $\left.c_{01}^{2}\right)^{2}$. Since, in view of $(2.5)$, the trace of $\tilde{h}_{q}$ is equal to 0 , the eigenvalues of $\tilde{h}_{q}$ are equal to $\pm \sqrt{-\left(c_{01}^{1}\right)^{2}+\frac{1}{4}\left(c_{02}^{1}-c_{01}^{2}\right)^{2}}$. We can choose

$$
\chi=-\left(c_{01}^{1}\right)^{2}+\frac{1}{4}\left(c_{02}^{1}-c_{01}^{2}\right)^{2}
$$

as a functional $t s$-invariant for our structure. In analogy with the sub-Riemannian case [1, 2, 7], we consider the functional $t s$-invariant defined as follows:

$$
\kappa=X_{2}\left(c_{12}^{1}\right)+X_{1}\left(c_{12}^{2}\right)-\left(c_{12}^{1}\right)^{2}+\left(c_{12}^{2}\right)^{2}-\frac{1}{2}\left(c_{01}^{2}+c_{02}^{1}\right) .
$$

Unlike the sub-Riemannian case where $\chi$ and $\kappa$ play the crucial role, it is $\tilde{h}$ and $\kappa$ that play the crucial role in the sub-Lorentzian setting.

Proposition 2.2. $\kappa$ is indeed a ts-invariant.

\subsubsection{Proof of Proposition 2.2}

Let $X_{1}, X_{2}$ is an orthonormal basis with a time orientation $X_{1}$ and a space orientation $X_{2}$, and let $c_{j k}^{i}$ be structures functions determined by this basis. Next, let $\theta=\theta(q)$ be a smooth functions and consider an orthonormal basis $Y_{1}, Y_{2}$ given by

$$
Y_{1}=X_{1} \cosh \theta+X_{2} \sinh \theta, \quad Y_{2}=X_{1} \sinh \theta+X_{2} \cosh \theta .
$$

Then $Y_{1}\left(Y_{2}\right)$ is a time (space) orientation, and of course

$$
X_{1}=Y_{1} \cosh \theta-Y_{2} \sinh \theta, \quad X_{2}=-Y_{1} \sinh \theta+Y_{2} \cosh \theta .
$$

Let $d_{j k}^{i}$ be the structure functions determined by the basis $Y_{1}, Y_{2}$, i.e.

$$
\begin{aligned}
& {\left[Y_{1}, Y_{0}\right]=d_{01}^{1} Y_{1}+d_{01}^{2} Y_{2}, \quad\left[Y_{2}, Y_{0}\right]=d_{02}^{1} Y_{1}+d_{02}^{2} Y_{2},} \\
& {\left[Y_{2}, Y_{1}\right]=d_{12}^{1} Y_{1}+d_{12}^{2} Y_{2}+X_{0} .}
\end{aligned}
$$

In order to prove Proposition 2.2 we need the following lemma.

Lemma 2.3. The following formulas hold true:

$$
\begin{aligned}
& d_{02}^{1}=-X_{0}(\theta)+c_{02}^{1} \cosh ^{2} \theta-c_{01}^{2} \sinh ^{2} \theta+\left(c_{01}^{1}-c_{02}^{2}\right) \sinh \theta \cosh \theta, \\
& d_{02}^{2}=\left(c_{01}^{2}-c_{02}^{1}\right) \sinh \theta \cosh \theta+c_{02}^{2} \cosh ^{2} \theta-c_{01}^{1} \sinh ^{2} \theta, \\
& d_{01}^{1}=c_{01}^{1} \cosh ^{2} \theta-c_{02}^{2} \sinh ^{2} \theta+\left(c_{02}^{1}-c_{01}^{2}\right) \sinh \theta \cosh \theta, \\
& d_{01}^{2}=-X_{0}(\theta)+c_{01}^{2} \cosh ^{2} \theta-c_{02}^{1} \sinh ^{2} \theta+\left(c_{02}^{2}-c_{01}^{1}\right) \sinh \theta \cosh \theta, \\
& d_{12}^{1}=\left(c_{12}^{1}-X_{1}(\theta)\right) \cosh \theta-\left(X_{2}(\theta)+c_{12}^{2}\right) \sinh \theta, \\
& d_{12}^{2}=\left(X_{1}(\theta)-c_{12}^{1}\right) \sinh \theta+\left(X_{2}(\theta)+c_{12}^{2}\right) \cosh \theta .
\end{aligned}
$$

Proof. All formulas are proved by direct calculations. For instance, using (2.8) we write

$$
\begin{aligned}
{\left[Y_{2}, Y_{1}\right] } & =\left[X_{1} \sinh \theta+X_{2} \cosh \theta, X_{1} \cosh \theta+X_{2} \sinh \theta\right] \\
& =-X_{1}(\theta) X_{1}+X_{2}(\theta) X_{2}+\left[X_{2}, X_{1}\right]
\end{aligned}
$$


Then using (2.8) and (2.3) we arrive at

$$
\begin{aligned}
& -X_{1}(\theta) X_{1}+X_{2}(\theta) X_{2}+c_{12}^{1} X_{1}+c_{12}^{2} X_{2}+X_{0} \\
& =-X_{1}(\theta)\left(Y_{1} \cosh \theta-Y_{2} \sinh \theta\right)+X_{2}(\theta)\left(-Y_{1} \sinh \theta+Y_{2} \cosh \theta\right) \\
& \quad+c_{12}^{1}\left(Y_{1} \cosh \theta-Y_{2} \sinh \theta\right)+c_{12}^{2}\left(-Y_{1} \sinh \theta+Y_{2} \cosh \theta\right)+X_{0}
\end{aligned}
$$

from which the fifth and sixth equations in (2.9) follow.

Now, using Lemma 2.3, we see that

$$
\frac{1}{2}\left(d_{01}^{2}+d_{02}^{1}\right)=-X_{0}(\theta)+\frac{1}{2}\left(c_{01}^{2}+c_{02}^{1}\right) .
$$

and

$$
\left(d_{12}^{1}\right)^{2}-\left(d_{12}^{2}\right)^{2}=\left(X_{1}(\theta)-c_{12}^{1}\right)^{2}-\left(X_{2}(\theta)+c_{12}^{2}\right)^{2} .
$$

Finally, we compute $Y_{2}\left(d_{12}^{1}\right)+Y_{1}\left(d_{12}^{2}\right)$. To this end let us write

$$
Y_{2}\left(d_{12}^{1}\right)+Y_{1}\left(d_{12}^{2}\right)=I+I I,
$$

where

$$
\begin{aligned}
I & =X_{2}\left(c_{12}^{1}\right)+X_{1}\left(c_{12}^{2}\right)-\left[X_{2}, X_{1}\right](\theta) \\
& =X_{2}\left(c_{12}^{1}\right)+X_{1}\left(c_{12}^{2}\right)-c_{12}^{1} X_{1}(\theta)-c_{12}^{2} X_{2}(\theta)-X_{0}(\theta)
\end{aligned}
$$

and

$$
I I=-c_{12}^{1} X_{1}(\theta)-X_{2}^{2}(\theta)-c_{12}^{2} X_{2}(\theta)+X_{1}^{2}(\theta) .
$$

Combining (2.10), (2.11) and (2.12) completes the proof of Proposition 2.2.

In summary, our basic $t s$-invariants are: a smooth function $\kappa$ on $M$ and a $(1,1)$ tensor $\tilde{h}$ on $H$.

\section{Sub-Lorentzian infinitesimal isometries and conformal transformations}

In this section $(M, H, g)$ is a fixed sub-semi-Riemannian manifold, $\operatorname{rank} H$ and $\operatorname{dim} M$ are arbitrary.

Definition 3.1. A a diffeomorphism $f: M \longrightarrow M$ is called a conformal transformation of $(M, H, g)$ if (i) $d_{q} f\left(H_{q}\right) \subseteq H_{f(q)}$ for every $q \in M$, (ii) there exists a function $\rho \in C^{\infty}(M), \rho>0$, such that

$$
g\left(d_{q} f(v), d_{q} f(w)\right)=\rho(q) g(v, w)
$$

for every $q \in M$ and every $v, w \in H_{q}$. If $\rho=1$ then $f$ is an isometry of $(M, H, g)$.

Along with conformal transformations and isometries we consider their infinitesimal variants.

Definition 3.2. A vector field $Z$ on $(M, H, g)$ is called an infinitesimal conformal transformation (resp. infinitesimal isometry) if its flow $\psi^{t}$ consists of conformal transformations (isometries).

Let us note a simple lemma. 
Lemma 3.3. Let $Z$ be a vector field on $M$ and denote by $\psi^{t}$ its flow. Then the following conditions are equivalent:

(a) $\operatorname{ad}_{Z}: \Gamma(H) \longrightarrow \Gamma(H)$;

(b) $d_{q} \psi^{t}: H_{q} \longrightarrow H_{\psi^{t}(q)}$ for every $q \in M$ and every $t$ such that $\psi^{t}$ is defined around $q$.

Proof. Although the result is known, we give a proof for the sake of completeness.

(a) $\Rightarrow$ (b) Following [23], we fix a point $q$ and consider a basis $X_{1}, \ldots, X_{k}$ of $H$ defined on a neighborhood $U$ of $q$. By our assumption, there exist smooth functions $\alpha_{i j}, i, j=1, \ldots, k$, such that $\operatorname{ad}_{Z} X_{i}=\sum_{j=1}^{k} \alpha_{i j} X_{j}$ on $U$ and it follows that if $v_{i}(t)=\left(\psi_{*}^{t} X_{i}\right)(q)=d \psi^{t} X_{i}\left(\psi^{-t} q\right)$ then

$$
\dot{v}_{i}(t)=\left(\psi_{*}^{t} \operatorname{ad}_{Z} X_{i}\right)(q)=\sum_{j=1}^{k}\left(\alpha_{i j} \circ \psi^{-t}\right)(q)\left(\psi_{*}^{t} X_{j}\right)(q)=\sum_{j=1}^{k} \beta_{i j}(t) v_{j}(t),
$$

where $\beta_{i j}(t)=\left(\alpha_{i j} \circ \psi^{-t}\right)(q)$. For any covector $\lambda \in T_{q}^{*} M$ which annihilates $H_{q}$, i.e., $\langle\lambda, v\rangle=0$ for every $v \in H_{q}$, we obtain a system of linear differential equations for the functions $w_{i}(t)=$ $\left\langle\lambda, v_{i}(t)\right\rangle, i=1, \ldots, k$ :

$$
\dot{w}_{i}(t)=\sum_{j=1}^{k} \beta_{i j}(t) w_{j}(t)
$$

with initial conditions $w_{i}(0)=0, i=1, \ldots, k$, since $v_{i}(0)=X_{i}(q) \in H_{q}$. Therefore $v_{i}(t)=0$ and $\left(\psi_{*}^{t} X_{i}\right)(q) \in H_{q}$ every $t$ for which $v_{i}(t)$ is defined, $i=1, \ldots, k$.

(b) $\Rightarrow$ (a) Take a point $q$, then for every $t$ such that $|t|$ is sufficiently small, we have $\left(\psi_{*}^{t} X\right)(q) \in$ $H_{q}$ and it follows that $\left(\operatorname{ad}_{Z} X\right)(q)=\left.\frac{d}{d t}\right|_{t=0}\left(\psi_{*}^{-t} X\right)(q) \in H_{q}$.

Suppose now that $f: M \longrightarrow M$ is a diffeomorphism such that $d f(H)=H$ and let $T$ be a tensor of type $(0,2)$ on $H$. We define a pull-back $\tilde{f}^{*}: \Gamma(H) \times \Gamma(H) \longrightarrow C^{\infty}(M)$ by

$$
\left(\tilde{f}^{*} T\right)_{q}(X, Y)=T_{f(q)}\left(d_{q} f(X), d_{q} f(Y)\right),
$$

where $X, Y \in \Gamma(H)$ (tilde indicates that we restrict to horizontal vector fields). We can now reformulate the definition of conformal transformations in a manner consistent with semiRiemannian geometry:

$f$ is a conformal transformation of $(M, H, g)$ if and only if there exists a function $\rho \in C^{\infty}(M)$, $\rho>0$, such that $\tilde{f}^{*} g=\rho g$ (if $\rho=1, f$ is an isometry).

Suppose that $Z$ is a vector field on $M$ such that $\operatorname{ad}_{Z}: \Gamma(H) \longrightarrow \Gamma(H)$ and let $\psi^{t}$ denote the (local) flow of $Z$. Using Lemma 3.3, again by analogy to the classical geometry, we can define a local operator $\tilde{L}_{Z} T: \Gamma(H) \times \Gamma(H) \longrightarrow C^{\infty}(M)$ which will be called the restricted Lie derivative:

$$
\left.\left(\tilde{L}_{Z} T\right)(q)=\left.\frac{d}{d t}\right|_{t=0}\left(\widetilde{\left(\psi^{t}\right.}\right)^{*} T\right)(q) .
$$

It turns out that

Proposition 3.4. A vector field $Z$ is an infinitesimal conformal transformation of $(M, H, g)$ if and only if the following conditions hold:

(i) $\operatorname{ad}_{Z}: \Gamma(H) \longrightarrow \Gamma(H)$, and

(ii) there exists a function $\mu \in C^{\infty}(M)$ such that $\tilde{L}_{Z} g=u g$. 
Proof. Remembering that we use only horizontal vector fields, the proof is the same as in the classical geometry. Again $\psi^{t}$ is the flow of $Z$.

" $\Rightarrow$ " By Lemma 3.3 we know that (i) is satisfied. If $\left(\widetilde{\psi^{t}}\right)^{*} g=\rho_{t} g$, where for each $t$ the function $\rho_{t}$ is smooth and positive, then it follows that

$$
\left.\left.\tilde{L}_{Z}\left(\left(\widetilde{\psi^{t}}\right)^{*} g\right)=\left.\frac{d}{d s}\right|_{s=0}\left(\widetilde{\psi^{s}}\right)^{*} \widetilde{\left(\psi^{t}\right.}\right)^{*} g=\frac{d}{d t} \widetilde{\left(\psi^{t}\right.}\right)^{*} g=\frac{d}{d t}\left(\rho_{t} g\right)=\dot{\rho}_{t} g .
$$

On the other hand, we also have that

$$
\left.\tilde{L}_{Z}\left(\widetilde{\psi^{t}}\right)^{*} g\right)=\tilde{L}_{Z}\left(\rho_{t} g\right)=X\left(\rho_{t}\right) g+\rho_{t}\left(\tilde{L}_{Z} g\right),
$$

and so we see that $\tilde{L}_{Z} g=\mu g$, where

$$
\mu=\frac{\dot{\rho}_{t}-X\left(\rho_{t}\right)}{\rho_{t}}
$$

(note that $\rho_{0}=1$ ).

" $\Leftarrow$ " From Lemma 3.3 we know that $d \psi^{t}$ preserves $H$. From (ii) and (3.1) we have

$$
\left.\left.\left.\frac{d}{d t}\left(\widetilde{\psi^{t}}\right)^{*} g=\widetilde{\left(\psi^{t}\right.}\right)^{*}\left(\tilde{L}_{Z} g\right)=\widetilde{\left(\psi^{t}\right.}\right)^{*}(\mu g)=\left(\mu \circ \psi^{t}\right) \widetilde{\left(\psi^{t}\right.}\right)^{*} g,
$$

which implies that $\left.\widetilde{\psi^{t}}\right)^{*} g=\rho_{t} g$, where

$$
\rho_{t}(q)=\exp \int_{0}^{t} \mu\left(\psi^{s}(q)\right) d s
$$

By direct calculation we obtain

$$
\left(\tilde{L}_{Z} g\right)(X, Y)=Z(g(X, Y))-g\left(\operatorname{ad}_{Z} X, Y\right)-g\left(X, \operatorname{ad}_{Z} Y\right)
$$

for every $X, Y \in \Gamma(H)$, which gives the following two corollaries:

Corollary 3.5. $Z$ is an infinitesimal conformal transformation of $(M, H, g)$ if and only if there exists a function $\mu \in C^{\infty}(M)$ such that for every $X, Y \in \Gamma(H)$

$$
Z(g(X, Y))=g\left(\operatorname{ad}_{Z} X, Y\right)+g\left(X, \operatorname{ad}_{Z} Y\right)+\mu g(X, Y) .
$$

Corollary 3.6. $Z$ is an infinitesimal isometry of $(M, H, g)$ if and only if for every $X, Y \in \Gamma(H)$

$$
Z(g(X, Y))=g\left(\operatorname{ad}_{Z} X, Y\right)+g\left(X, \operatorname{ad}_{Z} Y\right) .
$$

Furthermore:

Corollary 3.7. If $Z$ is an infinitesimal conformal transformation or isometry of $(M, H, g)$ then for every $n \geq 2$ and every $X, Y \in \Gamma(H)$

$$
\sum_{k=0}^{n}\left(\begin{array}{l}
n \\
k
\end{array}\right) g\left(\operatorname{ad}_{Z}^{k} X, \operatorname{ad}_{Z}^{n-k} Y\right)=0 .
$$

Proof. Fix a point $q \in M$. Under the above notation, for any $n \in \mathbb{N}$ and sufficiently small $|t|$

$$
\begin{array}{r}
\rho_{t}\left(\psi^{t} q\right) g\left(X\left(\psi^{t} q\right), Y\left(\psi^{t} q\right)\right)=g\left(d_{\psi^{t} q} \psi^{-t}(X), d_{\psi^{t} q} \psi^{-t}(Y)\right) \\
=g\left(\sum_{k=0}^{n} \frac{t^{k}}{k !}\left(\operatorname{ad}_{Z}^{k} X\right)(q), \sum_{m=0}^{n} \frac{t^{m}}{m !}\left(\operatorname{ad}_{Z}^{m} Y\right)(q)\right)+o\left(t^{n}\right) .
\end{array}
$$

Using Corollary 3.5 we can remove from (3.4) terms of order 0 and 1 with respect to $t$. What we obtain is

$$
\sum_{k=2}^{n} t^{k} \sum_{i+j=k} \frac{1}{i ! j !} g\left(\left(\operatorname{ad}_{Z}^{i} X\right)(q),\left(\operatorname{ad}_{Z}^{j} Y\right)(q)\right)+o\left(t^{n}\right)=0
$$

for $|t|$ sufficiently small, which gives (3.3). 


\section{Some properties of invariants}

In this section we assume all sub-Lorentzian manifolds to be $t s$-oriented. Let us start from an obvious observation.

Proposition 4.1. Let $\left(M_{i}, H_{i}, g_{i}\right), i=1,2$, be a contact 3 -dimensional ts-oriented sub-Lorentzian manifolds. Denote by $\chi_{i}, \kappa_{i}, \tilde{h}_{i}$ the corresponding objects defined by $\left(H_{i}, g_{i}\right), i=1,2$. If $\varphi:\left(M_{1}, H_{1}, g_{1}\right) \longrightarrow\left(M_{2}, H_{2}, g_{2}\right)$ is a local ts-isometry, then $\chi_{1}=\varphi^{*} \chi_{2}, \kappa_{1}=\varphi^{*} \kappa_{2}$, and $\tilde{h}_{1}=\varphi^{*} \tilde{h}_{2}$.

Fix a contact 3 -dimensional sub-Lorentzian manifold $(M, H, g)$. First of all let us notice how the invariant $\tilde{h}$ can be expressed in terms of the restricted Lie derivative of the metric $g$ in the direction of the Reeb field. Indeed, knowing (3.2) it is clear that for every $q \in M$ and every $v, w \in H_{q}$

$$
\bar{h}_{q}(v, w)=\frac{1}{2}\left(\tilde{L}_{X_{0}} g\right)(q)(v, w) .
$$

Such an approach allows to define higher-order invariants, namely those that correspond to the bilinear forms

$$
\bar{h}_{q}^{(l)}(v, w)=\frac{1}{2}\left(\tilde{L}_{X_{0}}^{l} g\right)(q)(v, w), \quad l=2,3, \ldots
$$

In this way, however, we will not obtain any formulas involving the structure functions $c_{12}^{i}$.

Using (4.1) we obtain the following proposition and corollary thereof.

Proposition 4.2. The Reeb vector field $X_{0}$ is an infinitesimal isometry for $(H, g)$ if and only if $\tilde{h}_{q}=0$ for every $q \in M$.

Corollary 4.3. If the Reeb vector field $X_{0}$ is an infinitesimal isometry for $(H, g)$ then $\chi=0$ everywhere.

Proposition 4.2 shows one of the ways how to produce sub-Lorentzian isometries. This is important because we know very little examples of such maps.

Next we study the effect on the invariants when we dilate the structure. To this end suppose that we have a sub-Lorentzian ts-oriented structure $(H, g)$ which is given by an orthonormal frame $X_{1}, X_{2}$ with a time (resp. space) orientation $X_{1}$ (resp. $X_{2}$ ). Let $s>0$ be a constant. Consider the sub-Lorentzian structure $\left(H^{\prime}, g^{\prime}\right)$ defined by assuming the frame $X_{1}^{\prime}=s X_{1}, X_{2}^{\prime}=$ $s X_{2}$ to be orthonormal with the time (resp. space) orientation $X_{1}^{\prime}$ (resp. $X_{2}^{\prime}$ ). The normalized one form $\omega^{\prime}$ which defines $H^{\prime}$ is given by $\omega^{\prime}=\frac{1}{s^{2}} \omega$, i.e., $d \omega^{\prime}\left(X_{1}^{\prime}, X_{2}^{\prime}\right)=\omega^{\prime}\left(\left[X_{2}^{\prime}, X_{1}^{\prime}\right]\right)=1$. It follows that the Reeb field is now $s^{2} X_{0}$. Then it is easy to see that (2.3) can be rewritten as

$$
\begin{aligned}
& {\left[X_{1}^{\prime}, X_{0}^{\prime}\right]=c_{01}^{\prime 1} X_{1}^{\prime}+c_{01}^{\prime 2} X_{2}^{\prime}, \quad\left[X_{2}^{\prime}, X_{0}^{\prime}\right]=c_{02}^{\prime 1} X_{1}^{\prime}+c_{02}^{\prime 2} X_{2}^{\prime},} \\
& {\left[X_{2}^{\prime}, X_{1}^{\prime}\right]=c_{12}^{\prime 1} X_{1}^{\prime}+c_{12}^{\prime 2} X_{2}^{\prime}+X_{0}^{\prime}}
\end{aligned}
$$

where $c_{j k}^{i}=s c_{j k}^{i}$. As a corollary we obtain

Proposition 4.4. Let $\chi, \kappa, \tilde{h}$ (resp. $\left.\chi^{\prime}, \kappa^{\prime}, \tilde{h}^{\prime}\right)$ be the ts-invariants of the sub-Lorentzian structure defined by an orthonormal basis $X_{1}, X_{2}$ (resp. by $X_{1}^{\prime}=s X_{1}, X_{2}^{\prime}=s X_{2}$ ). Then

$$
\chi^{\prime}=s^{2} \chi, \quad \kappa^{\prime}=s^{2} \kappa, \quad \tilde{h}^{\prime}=s \tilde{h} .
$$




\subsection{The case $\chi=0, \tilde{h} \neq 0$}

Next let us assume that $\chi(q)=0$ but $\tilde{h}_{q} \neq 0$ (i.e. $c_{01}^{1} \neq 0$ ) everywhere. As we shall see we are given an additional structure in this case. Indeed, the correspondence $q \longrightarrow \operatorname{ker} \tilde{h}_{q}$ defines an invariantly given field of directions. We can distinguish two cases: (i) $c_{01}^{1}=\frac{1}{2}\left(c_{02}^{1}-c_{01}^{2}\right)$, and (ii) $c_{01}^{1}=-\frac{1}{2}\left(c_{02}^{1}-c_{01}^{2}\right)$. In the first case the matrix of $\tilde{h}_{q}$ is of the form

$$
\left(\begin{array}{cc}
c_{01}^{1} & c_{01}^{1} \\
-c_{01}^{1} & -c_{01}^{1}
\end{array}\right)
$$

and $\operatorname{ker} \tilde{h}_{q}$ is spanned by $X_{1}(q)-X_{2}(q)$ for each $q$. In the second case the matrix of $\tilde{h}_{q}$ is equal to

$$
\left(\begin{array}{ll}
c_{01}^{1} & -c_{01}^{1} \\
c_{01}^{1} & -c_{01}^{1}
\end{array}\right)
$$

and $\operatorname{ker} \tilde{h}_{q}$ is spanned by $X_{1}(q)+X_{2}(q)$. Thus in the considered case there exists a line sub-bundle $L \longrightarrow M$ of $H$ on which $g$ is equal to zero. Of course this result is trivial under assumption on $t s$-orientation because then $H$ admits a global orthonormal basis $X_{1}, X_{2}$ and we have in fact two such subbundles, namely $\operatorname{Span}\left\{X_{1}+X_{2}\right\}$ and $\operatorname{Span}\left\{X_{1}-X_{2}\right\}$. What is interesting here is that the condition $\chi=0, \tilde{h} \neq 0$ does not depend on the assumption on orientation. Indeed, notice that if we change a time (resp. space) orientation keeping space (resp. time) one then $\tilde{h}$ is multiplied by -1 (because so is $X_{0}$ ). Moreover the condition $\chi=0, \tilde{h} \neq 0$ means that $\tilde{h}$ is a non-zero map with vanishing eigenvalues, the fact being independent of possible multiplication by -1 . Therefore the condition $\chi=0, \tilde{h} \neq 0$ makes sense even for an unoriented contact sub-Lorentzian structures. In this way we are led to the following proposition.

Proposition 4.5. Suppose that $(M, H, g)$ is a contact sub-Lorentzian manifold (we don't make any assumptions on orientation). If $\chi(q)=0$ and $\tilde{h}_{q} \neq 0$ for every $q \in M$ then there exists a line sub-bundle $L \longrightarrow M$ of $H$ on which $g$ is equal to zero.

Proof. Fix an arbitrary point $q \in M$. Let $Y_{1}, Y_{2}$ be an orthonormal basis for $(H, g)$ defined on a neighborhood $U$ of $q$, where $Y_{1}$ is timelike and $Y_{2}$ is spacelike. Supposing $Y_{1}$ (resp. $Y_{2}$ ) to be a time (resp. space) orientation we can apply the above construction of $t s$-invariants obtaining the corresponding objects $\chi_{U}$ and $\tilde{h}_{U}$. By our assumption and the above remark $\chi_{U}=0, \tilde{h}_{U} \neq 0$ on $U$, and we get an invariantly defined line sub-bundle $L_{U} \longrightarrow U: U \ni q \longrightarrow \operatorname{ker}\left(\tilde{h}_{U}\right)_{q}=$ : $L_{U}(q)$. We repeat the same construction around any point $q \in M$, which results in the family $\left\{L_{U} \longrightarrow U\right\}_{U \subset M}$ of line sub-bundles, indexed by elements $U$ of an open covering of $M$. By construction $L_{U}(q)=L_{U^{\prime}}(q)$ for any $q \in U \cap U^{\prime}$.

Let us note that if $M$ is simply connected, then the assertion of Proposition 4.5 holds true no matter the values of $\chi$ and $\tilde{h}$ are, because in this case the metric $(H, g)$ admits a global orthonormal frame, see [15].

\subsection{The case $\tilde{h}=0$}

We begin with the following proposition which is clear because $X_{0}$ is an infinitesimal isometry.

Proposition 4.6. If $\tilde{h}=0$ then $X_{0}(\kappa)=0$, i.e., $\kappa$ is constant along the trajectories of $X_{0}$.

By (2.7) the assumption $\tilde{h}=0$ implies

$$
c_{01}^{1}=c_{02}^{2}=0, \quad c_{02}^{1}=c_{01}^{2} .
$$


We will write $c=c_{02}^{1}=c_{01}^{2}$. Now (2.3) takes the form

$$
\left[X_{1}, X_{0}\right]=c X_{2}, \quad\left[X_{2}, X_{0}\right]=c X_{1}, \quad\left[X_{2}, X_{1}\right]=c_{12}^{1} X_{1}+c_{12}^{2} X_{2}+X_{0} .
$$

Rewriting as above (4.2) in terms of the dual forms $\nu_{i}$ we arrive at

$$
d \nu_{0}=\nu_{1} \wedge \nu_{2}, \quad d \nu_{1}=c \nu_{0} \wedge \nu_{2}+c_{12}^{1} \nu_{1} \wedge \nu_{2}, \quad d \nu_{2}=c \nu_{0} \wedge \nu_{1}+c_{12}^{2} \nu_{1} \wedge \nu_{2} .
$$

Lemma 4.7. The following identities hold

$$
-X_{1}(c)-c c_{12}^{2}+X_{0}\left(c_{12}^{1}\right)=0, \quad X_{2}(c)-c c_{12}^{1}+X_{0}\left(c_{12}^{2}\right)=0 .
$$

Proof. The lemma is obtained upon applying the exterior differential to both sides of the second and the third equation in (4.3).

Our next aim, which will be achieved in the next subsection, is to find a hyperbolic rotation of our frame $X_{1}, X_{2}$ so that (4.2) significantly simplifies. More precisely we want to kill the terms $c_{12}^{i}, i=1,2$. To this end let us introduce the following 1 -form

$$
\eta=(\kappa+c) \nu_{0}+c_{12}^{1} \nu_{1}-c_{12}^{2} \nu_{2}
$$

whose significance will become evident below.

Proposition 4.8. $d \eta=d \kappa \wedge \nu_{0}$.

Proof. Computations give

$$
\begin{aligned}
d \eta= & d \kappa \wedge \nu_{0}+\left(-X_{1}(c)-c c_{12}^{2}+X_{0}\left(c_{12}^{1}\right)\right) \nu_{0} \wedge \nu_{1}+\left(-X_{2}(c)+c c_{12}^{1}-X_{0}\left(c_{12}^{2}\right)\right) \nu_{0} \wedge \nu_{2} \\
& +\left(\kappa+c-X_{2}\left(c_{12}^{1}\right)-X_{1}\left(c_{12}^{2}\right)+\left(c_{12}^{1}\right)^{2}-\left(c_{12}^{2}\right)^{2}\right) \nu_{1} \wedge \nu_{2} .
\end{aligned}
$$

To end the proof we use Lemma 4.7 and the definition of $\kappa$.

\subsection{The simply-connected Lie group case}

Suppose that our contact sub-Lorentzian manifold $(M, H, g)$ is such that $M$ is a simply-connected Lie group and $H, g$ are left-invariant; this means that left translations of $M$ are sub-Lorentzian isometries (note that any left-invariant bracket generating distribution on a 3-dimensional Lie group is necessarily contact). In such a case, clearly, $\chi$ and $\kappa$ are constant. We also remark that unlike the general situation, the assumption on $t s$-orientation is no longer restrictive since groups are parallelizable manifolds. As above, assume that $\tilde{h}=0$ everywhere.

Recalling our aim formulated in the previous subsection we prove the following lemma.

Lemma 4.9. There exists a smooth function $\theta: M \longrightarrow \mathbb{R}$ such that $X_{1}(\theta)=c_{12}^{1}, X_{2}(\theta)=-c_{12}^{2}$.

Proof. Suppose that such a function $\theta$ exists. Then

$$
\begin{aligned}
X_{0}(\theta) & =\left[X_{2}, X_{1}\right](\theta)-c_{12}^{1} X_{1}(\theta)-c_{12}^{2} X_{2}(\theta) \\
& =X_{2}\left(c_{12}^{1}\right)+X_{1}\left(c_{12}^{2}\right)-\left(c_{12}^{1}\right)^{2}+\left(c_{12}^{2}\right)^{2}=\kappa+c,
\end{aligned}
$$

and it follows that

$$
d \theta=X_{0}(\theta) \nu_{0}+X_{1}(\theta) \nu_{1}+X_{2}(\theta) \nu_{2}=(\kappa+c) \nu_{0}+c_{12}^{1} \nu_{1}-c_{12}^{2} \nu_{2}=\eta
$$

where $\eta$ is defined by (4.4). Thus to prove the existence of $\theta$ it is enough to show that $\eta$ is exact. Since $M$ is simply-connected we must show that $d \eta=0$. This is however clear by Proposition 4.8 and the fact that $\kappa$ is a constant. 
Now we apply to our frame $X_{1}, X_{2}$, the hyperbolic rotation by the angle $\theta$ specified above. As a result, the frame $Y_{1}, Y_{2}$ given by (2.8) satisfies

\section{Proposition 4.10.}

$$
\left[Y_{1}, X_{0}\right]=-\kappa Y_{2}, \quad\left[Y_{2}, X_{0}\right]=-\kappa Y_{1}, \quad\left[Y_{2}, Y_{1}\right]=X_{0}
$$

Proof. It follows directly from facts proved in Subsections 4.2, 4.3, from (4.2), and from Lemma 2.3.

We observe here the difference between the sub-Riemannian case where the brackets have the form

$$
\left[Y_{1}, X_{0}\right]=\kappa Y_{2}, \quad\left[Y_{2}, X_{0}\right]=-\kappa Y_{1}, \quad\left[Y_{2}, Y_{1}\right]=X_{0}
$$

We remark that in the sub-Riemannian case Agrachev and Barilari [1] obtain the following results: $\kappa=0$ implies $M$ is isometric to the Heisenberg group, $\kappa>0$ implies $M$ is isometric to $\mathrm{SU}_{2}$ and $\kappa<0$ implies $M$ is isometric to the universal cover of $\mathrm{SL}_{2}$. In the sub-Lorentzian case $\mathrm{SU}_{2}$ does not arise. To be precise we have the following corollaries of Proposition 4.10.

Corollary 4.11. If $M$ is a simply-connected Lie group such that $\tilde{h}$ and $\kappa$ vanish identically, then $M$ is isometric to the Heisenberg group.

Corollary 4.12. If $M$ is a simply-connected Lie group such that $\tilde{h}$ vanishes and $\kappa \neq 0$, then it is isometric to a sub-Lorentzian structure on $\widetilde{\mathrm{SL}_{2}}(\mathbb{R})$ induced by the Killing form.

Before proving Corollary 4.12 let us recall some basic facts about the Killing form and Cartan decompositions. For any Lie algebra $\mathfrak{g}$ the Killing form is the symmetric bilinear form defined by $K(X, Y)=\operatorname{Trace}\left(\operatorname{ad}_{X} \operatorname{ad}_{Y}\right)$. The Killing form has the following invariance properties:

1) $K([X, Y], Z)=K(X,[Y, Z])$,

2) $K(T(X), T(Y))=K(X, Y)$ for all $T \in \operatorname{Aut}(\mathfrak{g})$.

If $\mathfrak{g}$ is simple then any symmetric bilinear form satisfying the first invariance condition is a scalar multiple of the Killing form and Cartan's criterion states that a Lie algebra is semisimple if and only if the Killing form is non-degenerate.

A Cartan involution is any element $\Theta \in \operatorname{Aut}(\mathfrak{g})$ such that $\Theta^{2}=I$ and

$$
\langle X, Y\rangle_{\Theta}=-K(X, \Theta(Y))
$$

is positive definite. Corresponding with $\Theta$ we have a Cartan decomposition $\mathfrak{g}=\mathfrak{t} \oplus \mathfrak{p}$, where $\mathfrak{t}$ and $\mathfrak{p}$ are the eigenspaces corresponding with the eigenvalues 1 and -1 respectively. Since $\Theta$ is an automorphism, it follows that $[\mathfrak{t}, \mathfrak{t}] \subseteq \mathfrak{t},[\mathfrak{t}, \mathfrak{p}] \subseteq \mathfrak{p}$ and $[\mathfrak{p}, \mathfrak{p}] \subseteq \mathfrak{t}$. Moreover, the Killing form is negative definite on $\mathfrak{t}$ and positive definite on $\mathfrak{p}$.

The standard Cartan involution on $\mathfrak{s l}_{2}$ is given by $\Theta(A)=-A^{T}$. In this case we have that $\mathfrak{t}=\operatorname{span}\left\{f_{1}\right\}$ and $\mathfrak{p}=\operatorname{span}\left\{f_{2}, f_{0}\right\}$, where

$$
f_{0}=\frac{1}{2}\left(\begin{array}{cc}
-1 & 0 \\
0 & 1
\end{array}\right), \quad f_{1}=\frac{1}{2}\left(\begin{array}{cc}
0 & 1 \\
-1 & 0
\end{array}\right), \quad f_{2}=\frac{1}{2}\left(\begin{array}{ll}
0 & 1 \\
1 & 0
\end{array}\right),
$$

and the Lie brackets are

$$
\left[f_{2}, f_{1}\right]=f_{0}, \quad\left[f_{1}, f_{0}\right]=f_{2}, \quad\left[f_{2}, f_{0}\right]=f_{1}
$$


The Killing form for $\mathfrak{s l}_{2}$ is given by $K(A, B)=4 \operatorname{Tr}(A B)$ and so the bilinear form $B(A, B)=$ $\frac{1}{2} K(A, B)$ satisfies

$$
\begin{array}{lll}
B\left(f_{0}, f_{0}\right)=1, & B\left(f_{1}, f_{1}\right)=-1, & B\left(f_{2}, f_{2}\right)=1, \\
B\left(f_{0}, f_{1}\right)=0, & B\left(f_{0}, f_{2}\right)=0, & B\left(f_{1}, f_{2}\right)=0 .
\end{array}
$$

Thus we have two choices: 1) $\mathcal{H}_{e}=\operatorname{span}\left\{f_{1}, f_{2}\right\}$ or 2) $\mathcal{H}_{e}=\operatorname{span}\left\{f_{1}, f_{0}\right\}$. In each case, by left translation, we obtain left-invariant sub-Lorentzian structures on $\widetilde{\mathrm{SL}}_{2}(\mathbb{R})$ satisfying $\tilde{h}=0$. An isometry between these two structures is induced by Lie algebra automorphism $T$, where $T f_{0}=f_{2}, T f_{1}=-f_{1}$ and $T f_{2}=f_{0}$.

Proof of Corollary 4.12. First we observe that the matrices

$$
e_{0}=\frac{1}{2}\left(\begin{array}{cc}
\kappa & 0 \\
0 & -\kappa
\end{array}\right), \quad e_{1}=\frac{1}{2}\left(\begin{array}{cc}
0 & 1 \\
\kappa & 0
\end{array}\right), \quad e_{2}=\frac{1}{2}\left(\begin{array}{cc}
0 & 1 \\
-\kappa & 0
\end{array}\right)
$$

form a basis of $\mathfrak{s l}_{2}$ and the bracket relations are $\left[e_{2}, e_{1}\right]=e_{0},\left[e_{1}, e_{0}\right]=-\kappa e_{2},\left[e_{2}, e_{0}\right]=-\kappa e_{1}$. Furthermore $K\left(e_{1}, e_{1}\right)=2 \kappa, K\left(e_{2}, e_{2}\right)=-2 \kappa$, and $K\left(e_{1}, e_{2}\right)=0$. Since we assume the subLorentzian structure on $M$ is left-invariant, the metric must be the left translation of the metric $B(A, B)=-\frac{1}{2 \kappa} K(A, B)$ on $T_{e} M=\mathfrak{s l}_{2}$. Since $M$ is simply connected it must be the universal cover of $\mathrm{SL}_{2}(\mathbb{R})$.

We remark that in general

$$
\begin{array}{lll}
K\left(e_{0}, e_{0}\right)=2 \kappa^{2}, & K\left(e_{1}, e_{1}\right)=2 \kappa, & K\left(e_{2}, e_{2}\right)=-2 \kappa, \\
K\left(e_{0}, e_{1}\right)=0, & K\left(e_{0}, e_{2}\right)=0, & K\left(e_{1}, e_{2}\right)=0,
\end{array}
$$

and so the corresponding Cartan involution is given by

$$
\Theta\left(e_{0}\right)=-e_{0}, \quad \Theta\left(e_{1}\right)=-e_{1}, \quad \Theta\left(e_{2}\right)=e_{2} .
$$

Hence $\mathfrak{t}=\operatorname{span}\left\{e_{2}\right\}$ and $\mathfrak{p}=\operatorname{span}\left\{e_{1}, e_{0}\right\}$. If $|\kappa| \neq 1$, then since $K\left(e_{0}, e_{0}\right)=2 \kappa^{2}$, the only choice we have for a sub-Lorentzian structure induced by the Killing form is $\mathcal{H}_{e}=\operatorname{span}\left\{e_{1}, e_{2}\right\}$.

The null lines in $\mathfrak{s l}_{2}$ are $\operatorname{span}\left\{e_{1}-e_{2}\right\}$ and $\operatorname{span}\left\{e_{1}+e_{2}\right\}$. Furthermore if we set

$$
n_{0}=e_{3}, \quad n_{1}=\frac{1}{\sqrt{2}}\left(e_{1}-e_{2}\right), \quad n_{2}=\frac{1}{\sqrt{2}}\left(e_{1}+e_{2}\right)
$$

then

$$
\left[n_{2}, n_{1}\right]=n_{0}, \quad\left[n_{1}, n_{0}\right]=\kappa n_{1}, \quad\left[n_{2}, n_{0}\right]=-\kappa n_{2}
$$

If we set $\mathcal{H}_{e}=\operatorname{span}\left\{n_{1}, n_{2}\right\}$ and define

$$
B\left(n_{1}, n_{1}\right)=-1, \quad B\left(n_{2}, n_{2}\right)=1, \quad B\left(n_{1}, n_{2}\right)=0,
$$

then the induced left-invariant structure on $\widetilde{\mathrm{SL}}_{2}(\mathbb{R})$ is isometrically distinct from the cases above, indeed

$$
\tilde{h}=\kappa\left(\begin{array}{cc}
1 & 0 \\
0 & -1
\end{array}\right) .
$$




\section{Infinitesimal sub-Lorentzian transformations on groups with $\tilde{h}=0$}

\subsection{Introduction}

In this section we determine the conformal and isometry groups for the Heisenberg group and the universal cover of $\mathrm{SL}_{2}$. In particular we will see that in both cases the local infinitesimal conformal transformations are given by $\mathfrak{s l}_{3}$.

In the context of this paper it would be natural to construct the vector fields using the criteria developed in Section 3, however this leads to complicated systems of PDEs which we cannot provide explicit proof concerning solutions. Instead we apply Cartan's equivalence method which leads to the general solution without having to solve PDEs.

In work in preparation with Alexandr Medvedev [16] we explore further the application of the Cartan approach to sub-Lorentzian geometry. In particular the invariants discussed here appear in a much more systematic manner and a complete description of all left-invariant sub-Lorentzian structures on 3-dimensional Lie groups will be given.

We also remark that on the subject of conformal classification of left-invariant sub-Riemannian structures on 3-dimensional Lie groups there is the very recent arXiv paper of Boarotto [5].

\subsection{Preliminaries}

The assumption $\tilde{h}=0$ implies that the Lie algebra of $M$ has the form given in Proposition 4.10. So as to make the notation a little less confusing in the calculations that ensue, we rewrite the Lie algebra in the following form

$$
\left[X_{1}, X_{3}\right]=\kappa X_{2}, \quad\left[X_{2}, X_{3}\right]=\kappa X_{1}, \quad\left[X_{1}, X_{2}\right]=X_{3} .
$$

We denote the ordered dual frame by $\theta=\left\{\theta_{1}, \theta_{2}, \theta_{3}\right\}$ and observe that by Cartan's formula the structure equations of this coframe are

$$
d \theta_{1}=\kappa \theta_{3} \wedge \theta_{2}, \quad d \theta_{2}=\kappa \theta_{3} \wedge \theta_{1}, \quad d \theta_{0}=\theta_{2} \wedge \theta_{1} .
$$

Moreover the sub-Lorentzian metric has the form $\theta_{2} \odot \theta_{2}-\theta_{1} \odot \theta_{1}$. It follows that the subgroup of $G \subset G L(3)$ which acts on $\theta$ and leaves the metric conformally invariant modulo terms of the form $\eta \odot \theta_{3}$, consists of matrices of the form

$$
\left(\begin{array}{ccc}
e^{r} \cosh (t) & e^{r} \sinh (t) & a \\
e^{r} \sinh (t) & e^{r} \cosh (t) & b \\
0 & 0 & e^{2 r}
\end{array}\right) .
$$

Hence a local diffeomorphism $f: M \rightarrow M$ is conformal if and only if

$$
f^{*} \theta=g_{f} \theta,
$$

where $g_{f}: M \rightarrow G$. Thus the conformal symmetry problem is exactly to find all local diffeomorphisms which satisfy (5.1) which is precisely a Cartan equivalence problem.

\subsection{Overview of Cartan's algorithm}

For the details we refer the reader to [24]. The first step in Cartan's algorithm is to pass to the equation $f^{*} d \theta=d\left(g_{f} \theta\right)$ and lift the problem to $M \times G$. In this context the lift of $\theta$ is given by the partial coframe $\Theta \subset T^{*}(M \times G)$, where $\Theta_{(p, g)}=g \theta_{p}$. The lift of $f$ is defined by $\tilde{f}(p, g)=\left(f(p), g g_{f}(p)^{-1}\right)$ and it follows that

$$
\tilde{f}^{*} \Theta=\Theta \quad \text { and } \quad \tilde{f}^{*} d \Theta=d \Theta .
$$


If $\Pi \subset T(M \times G)^{*}$ is any subset complementary to $\Theta$ then the structure equations for the lifted partial coframe take the form:

$$
d \Theta=\Pi \wedge \Theta+T \Theta \wedge \Theta
$$

and $T$ is referred to as the torsion. It follows from (5.2) that if $f$ is a local conformal diffeomorphism then $T \circ \tilde{f}=T$, however in general this equation is not the end of the story. Roughly speaking all the higher-order coframe derivatives of $T$ must also be invariant under composition with $\tilde{f}$, see [24, Theorem 14.24] for a precise statement. Fortunately in our case this will not be an issue.

The main idea in the Cartan algorithm is to exploit the freedom of choice of $\Pi$ so as to minimise the torsion $T$. There are three processes involved in minimising torsion, namely group reduction, absorption and prolongation. A reduction of the structure group can be carried out when the condition $f^{*} \theta=g_{f} \theta$ for some $g_{f}: M \rightarrow G$ implies that $g_{f}: M \rightarrow G^{\prime} \subset G$. Reductions reveal themselves as coefficients in $T$ depending only on group parameters. Such coefficients can be set to a convenient constant value as long as invertibilty is not violated. An example of such a phenomena that the reader may be familiar with occurs in the contact equivalence problem in dimension 3, i.e., if initially we assume that $g=\left(g_{i j}\right)$ and $g_{31}=g_{32}=0$ then it transpires that $g_{33}=g_{11} g_{22}-g_{12} g_{21}$.

Absorption utilises the fact, that pointwise, each element of $\Pi$ is a Maurer-Cartan form on $G$ plus a linear combination of the $\Theta_{i}$. We choose the coefficients of the $\Theta_{i}$ so that $T$ has as many zero coefficients as possible.

In summary the element $T_{i j}^{k} \in T$ is the coefficient of $\Theta^{i} \wedge \Theta^{j}$ in the expression for $d \Theta^{k}$. If $T_{i j}^{k}$ is independent of absorption parameters then it gives a group reduction by setting it equal to \pm 1 or 0 and the choice is made so as not to violate invertibilty. If $T_{i j}^{k}$ is dependent on absorption parameters then we solve one of these parameters so that $T_{i j}^{k}=0$. The aim of the algorithm is to reduce the group to $\{\mathrm{Id}\}$ through a sequence of reduction and absorption cycles.

If after the first reduction and absorption we get $G=\operatorname{Id}$ then the resulting $T$ consists of the basic invariants for the equivalence problem. Otherwise not all group parameters are normalised and absorption parameters may remain undetermined with no torsion coefficients available to normalise them. In this case the problem must be prolonged which means that the free absorption parameters are understood as the groups parameters for a structure group $G^{(1)}$ associated with a new equivalence problem on $M \times G$. Specifically we write $\Pi=\varpi+F \Theta$, where $F$ consists of the free absorption parameters, and consider the equivalence problem for the partial coframe $\Theta \cup \varpi \subset T^{*}(M \times G)$ with structure group $G^{(1)}$ consisting of matrices of the form

$$
\left(\begin{array}{ll}
I & 0 \\
F & I
\end{array}\right)
$$

In this context $\Theta \cup \Pi \subset T^{*}\left(M \times G \times G^{(1)}\right)$ is a lift of $\Theta \cup \varpi$ and we repeat the procedure: augment, reduce and absorb ..., until eventually we get an equivalence problem where the structure group reduces to the identity and all absorption coefficients are determined. Of course it can happen that the process will not lead to such a situation, but when it does, the result is the structure equations of a certain Cartan connection on $M$.

\subsection{Calculations}

To begin we lift and define one forms $\Theta_{i}$ on $M \times G$ by setting

$$
\left(\begin{array}{c}
\Theta_{1} \\
\Theta_{2} \\
\Theta_{3}
\end{array}\right)=\left(\begin{array}{ccc}
e^{r} \cosh (t) & e^{r} \sinh (t) & a \\
e^{r} \sinh (t) & e^{r} \cosh (t) & b \\
0 & 0 & e^{2 r}
\end{array}\right)\left(\begin{array}{c}
\theta_{1} \\
\theta_{2} \\
\theta_{3}
\end{array}\right)
$$


and augment the set $\left\{\Theta_{1}, \Theta_{2}, \Theta_{3}\right\} \subset T^{*}(M \times G)$ with the following forms:

$$
\begin{aligned}
& \Pi_{1}=\alpha_{1}+\frac{1}{2} b e^{-2 r} \Theta_{1}-\frac{1}{2} a e^{-2 r} \Theta_{2}-\left(B_{1}+a b e^{-4 r}\right) \Theta_{3}, \\
& \Pi_{2}=\alpha_{2}-\frac{3}{2} a e^{-2 r} \Theta_{1}+\frac{3}{2} b e^{-2 r} \Theta_{2}+\left(a^{2} e^{-4 r}+\kappa e^{-2 r}-B_{2}\right) \Theta_{3}, \\
& \Pi_{3}=\alpha_{3}-B_{1} \Theta_{1}-B_{2} \Theta_{2}-B_{3} \Theta_{3}, \\
& \Pi_{4}=\alpha_{4}+\left(\left(a^{2}+b^{2}\right) e^{-4 r}-B_{2}\right) \Theta_{1}-\left(2 a b e^{-4 r}+B_{1}\right) \Theta_{2}-B_{4} \Theta_{3} .
\end{aligned}
$$

The coefficients of the $\Theta_{i}$ in $\Pi_{j}$ are determined by absorbing torsion and the $\alpha_{j}$ are the Maurer-Cartan forms:

$$
\alpha_{1}=d r, \quad \alpha_{2}=d t, \quad \alpha_{3}=(d a-a d r-b d t) e^{-2 r}, \quad \alpha_{4}=(d b-b d r-a d t) e^{-2 r} .
$$

The coefficients $B_{1}, \ldots, B_{4}$ are undetermined parameters from absorption and so a prolongation is required. We write

$$
\begin{aligned}
& \Pi_{1}=\varpi_{1}-B_{1} \Theta_{3}, \quad \Pi_{2}=\varpi_{2}-B_{2} \Theta_{3}, \quad \Pi_{3}=\varpi_{3}-B_{1} \Theta_{1}-B_{2} \Theta_{2}-B_{3} \Theta_{3}, \\
& \Pi_{4}=\varpi_{4}-B_{2} \Theta_{1}-B_{1} \Theta_{2}-B_{4} \Theta_{3} .
\end{aligned}
$$

and consider the equivalence problem $M \times G$ given by the ordered basis

$$
\left\{\Theta_{1}, \Theta_{2}, \Theta_{3}, \varpi_{1}, \varpi_{2}, \varpi_{3}, \varpi_{4}\right\}
$$

with structure group $G^{(1)}$ consisting of matrices of the form

$$
\left(\begin{array}{ll}
I & 0 \\
R & I
\end{array}\right) \text {, where } \quad R=\left(\begin{array}{ccc}
0 & 0 & -B_{1} \\
0 & 0 & -B_{2} \\
-B_{1} & -B_{2} & -B_{3} \\
-B_{2} & -B_{1} & -B_{4}
\end{array}\right) \text {. }
$$

The ordered basis $\left\{\Theta_{1}, \Theta_{2}, \Theta_{3}, \Pi_{1}, \Pi_{2}, \Pi_{3}, \Pi_{4}\right\}$ is now viewed as the lift of (5.3) to the 11dimensional manifold $M \times G \times G^{(1)}$ and again is augmented by forms $\left\{\Omega_{1}, \Omega_{2}, \Omega_{3}, \Omega_{4}\right\} \subset T^{*}(M \times$ $\left.G \times G^{(1)}\right)$. We get the following reductions of the structure group $G^{(1)}$ :

$$
\begin{aligned}
& B_{2}=\frac{1}{4} a^{2} e^{-4 r}+\frac{3}{4} b^{2} e^{-4 r}+\frac{1}{4} e^{-2 r} \kappa, \quad B_{3}=\frac{1}{2} b\left(\left(a^{2}-b^{2}\right) e^{-6 r}+e^{-4 r} \kappa\right), \\
& B_{4}=\frac{1}{2} a\left(\left(a^{2}-b^{2}\right) e^{-6 r}+e^{-4 r} \kappa\right)
\end{aligned}
$$

and so $M \times G \times G^{(1)}$ becomes an 8-dimensional manifold and we only need $\Omega=\Omega_{4}$ to augment.

Finally after absorption we arrive at the structure equations:

$$
\begin{aligned}
d \Theta_{1} & =\Pi_{1} \wedge \Theta_{1}+\Pi_{2} \wedge \Theta_{2}+\Pi_{3} \wedge \Theta_{3}, \\
d \Theta_{2} & =\Pi_{1} \wedge \Theta_{2}+\Pi_{2} \wedge \Theta_{1}+\Pi_{4} \wedge \Theta_{3}, \\
d \Theta_{3} & =2 \Pi_{1} \wedge \Theta_{3}-\Theta_{1} \wedge \Theta_{2}, \\
d \Pi_{1} & =\frac{1}{2} \Pi_{4} \wedge \Theta_{1}-\frac{1}{2} \Pi_{3} \wedge \Theta_{2}-\Omega \wedge \Theta_{3}, \\
d \Pi_{2} & =\frac{3}{2} \Pi_{4} \wedge \Theta_{2}-\frac{3}{2} \Pi_{3} \wedge \Theta_{1}, \\
d \Pi_{3} & =\Pi_{3} \wedge \Pi_{1}-\Pi_{4} \wedge \Pi_{2}-\Omega \wedge \Theta_{1}, \\
d \Pi_{4} & =\Pi_{4} \wedge \Pi_{1}-\Pi_{3} \wedge \Pi_{2}-\Omega \wedge \Theta_{2},
\end{aligned}
$$




$$
d \Omega=2 \Omega \wedge \Pi_{1}-\Pi_{4} \wedge \Pi_{3}
$$

The structure equations for the isometries are obtained similarly but do not require prolongation. The structure group is as above except $r=0$ and the structure equations are:

$$
d \Theta_{1}=\Pi \wedge \Theta_{2}, \quad d \Theta_{2}=\Pi \wedge \Theta_{1}, \quad d \Theta_{3}=\Theta_{2} \wedge \Theta_{1}, \quad d \Pi=\kappa \Theta_{2} \wedge \Theta_{1} .
$$

In both sets of structure equations the coefficients are all constant and as a consequence the symmetries of these structure equations are given by the Lie group which they define (see the remarks following [24, Theorem 8.22]). By construction the lift of our original symmetry is a symmetry of the structure equations and must therefore be given by the action of the Lie group which the structure equations define.

From (5.5) we see that the isometries are at most a 4-dimensional Lie group. By Tanaka's theory [25], the maximal dimension is reached when $\kappa=0$ and the structure equations are those of the Heisenberg group extended by the action of a particular strata preserving derivation of the Heisenberg algebra. The Lie algebra of this group has the form

$$
\left[e_{1}, e_{2}\right]=e_{3}, \quad\left[e_{4}, e_{1}\right]=e_{2}, \quad\left[e_{4}, e_{2}\right]=e_{1},
$$

where $\left\{e_{1}, e_{2}, e_{3}\right\}$ is a basis for the Heisenberg algebra and $e_{4}$ is the derivation.

The Killing form for the conformal structure equations is

$$
K=\left(\begin{array}{cccccccc}
0 & 0 & 0 & 0 & 0 & 0 & -7 & 0 \\
0 & 0 & 0 & 0 & 0 & 6 & 0 & 0 \\
0 & 0 & 0 & 0 & 0 & 0 & 0 & 6 \\
0 & 0 & 0 & 12 & 0 & 0 & 0 & 0 \\
0 & 0 & 0 & 0 & 4 & 0 & 0 & 0 \\
0 & 6 & 0 & 0 & 0 & 0 & 0 & 0 \\
-7 & 0 & 0 & 0 & 0 & 0 & 0 & 0 \\
0 & 0 & 6 & 0 & 0 & 0 & 0 & 0
\end{array}\right)
$$

Since $\operatorname{det} K \neq 0(=-3048192)$ and $K$ is indefinite with signature ++++--- , the Lie algebra must be $\mathfrak{s l}_{3}$, i.e., the only 8-dimensional simple Lie algebras are $\mathfrak{s l}_{3}, \mathfrak{s u}_{3}$ and $\mathfrak{s u}_{2,1}$, however $\mathfrak{s u}_{3}$ and $\mathfrak{s u}_{2,1}$ are ruled out by the indefiniteness and signature. Alternatively one can simply compute the Lie brackets of the vector fields dual to the system of one forms and check that it isomorphic to $\mathfrak{s l}_{3}$. We should remark that Ian Anderson's MAPLE DifferentialGeometry packages were an indispensable tool used in the calculations outlined above.

The fact that $\kappa$ is not present in (5.4) implies that the universal cover of $\mathrm{SL}_{2}(\mathbb{R})$ and the Heisenberg group both have $\mathrm{SL}_{3}(\mathbb{R})$ as the conformal symmetry group and consequently are conformally equivalent, see [22, Proposition 2.3.2] and [6, Section 2.5]. We thus have the following conformal Darboux theorem.

Theorem 5.1. All left-invariant sub-Lorentzian structures on the universal cover of $\mathrm{SL}_{2}(\mathbb{R})$ such that $\tilde{h}=0$ are locally conformally equivalent to the sub-Lorentzian Heisenberg group.

\section{Rigid example}

By definition, any left translation is an isometry of a left-invariant structure and so the dimension of the isometry group is at least 3 , and from the previous section the dimension of the isometry group is at most 4 . The goal of this section is to show that the extreme case of no isometries can occur but obviously not for a left invariant structure. A relatively straight forward example comes from the geometry of second-order ODEs. 
To a given second-order ODE

$$
u^{\prime \prime}=Q\left(x, u, u^{\prime}\right)
$$

where $Q$ is smooth, we associate three 1 -forms given by

$$
\omega^{1}=d u-p d x, \quad \omega^{2}=d p-Q(x, u, p) d x, \quad \omega^{3}=d x,
$$

which are regarded as one forms jet space $J^{1}(\mathbb{R}, \mathbb{R})$ with coordinates by $(x, u, p)$, where $x$ is the independent variable, $u$ is the dependent variable and $p=u^{\prime}$, see [24].

In particular a curve $\gamma(x)=(x, u(x), p(x))$ in $J^{1}(\mathbb{R}, \mathbb{R})$ defines a solution to (6.1) if and only if $\gamma^{*} \omega^{i}=0, i=1,2$ (one can easily show that the vanishing of the two pull-backs is equivalent to $y^{\prime}(x)=p(x)$ and in turn to $\left.u^{\prime \prime}(x)=Q\left(x, u(x), u^{\prime}(x)\right)\right)$. A local diffeomorphism $\Phi: \mathbb{R} \times \mathbb{R} \longrightarrow \mathbb{R} \times \mathbb{R}$ is called a point transformation or point symmetry of (6.1) if and only if it maps the graph of a solutions to (6.1) onto graphs of solutions to (6.1).

Any local diffeomorphism $\Phi: \mathbb{R} \times \mathbb{R} \longrightarrow \mathbb{R} \times \mathbb{R}$ can always be prolonged to a local diffeomorphism $\hat{\Phi}: J^{1}(\mathbb{R}, \mathbb{R}) \rightarrow J^{1}(\mathbb{R}, \mathbb{R})$ by setting $\hat{\Phi}(x, u, p)=(\tilde{x}, \tilde{u}, \tilde{p})$, where

$$
(\tilde{x}, \tilde{u})=\Phi(x, u) \quad \text { and } \quad \tilde{p}=\frac{d \tilde{u}}{d \tilde{x}} .
$$

By direct calculation it follows that $\Phi$ is a point symmetry of (6.1) if and only if there exists smooth functions $a_{i}, i=1, \ldots, 5$, such that

$$
\hat{\Phi}^{*} \omega^{1}=a_{1} \omega^{1}, \quad \hat{\Phi}^{*} \omega^{2}=a_{2} \omega^{1}+a_{3} \omega^{2}, \quad \hat{\Phi}^{*} \omega^{3}=a_{4} \omega^{1}+a_{5} \omega^{3} .
$$

A classical problem in the geometric theory of ODEs is the classification of second-order ODEs with respect to point transformations. The fundamental result is as follows (see [24, Theorem 12.19]):

Theorem 6.1. The point transformation symmetry group of a second-order ordinary differential equation has dimension at most eight. Moreover, the equation admits an eight-dimensional symmetry group if and only if it can be mapped by a point transformation to the linear equation $u^{\prime \prime}=0$, which has symmetry group $\mathrm{SL}(3)$.

Thus the equation $u^{\prime \prime}=0$ has the maximal possible point symmetry group while at the other end of the scale the following equation

$$
u^{\prime \prime}=\left(\left(x+x^{2}\right) e^{u}\right)^{\prime}
$$

has no nontrivial point symmetries (see [24, p. 182]).

Any equation (6.1) defines through the forms (6.2) a conformal class of contact sub-Lorentzian metrics. Indeed, let $H=\operatorname{ker} \omega^{1}, L_{1}=\operatorname{ker} \omega^{1} \cap \operatorname{ker} \omega^{2}$ and $L_{2}=\operatorname{ker} \omega^{1} \cap \operatorname{ker} \omega^{3}$. Clearly, $H$ is a contact distribution that splits into the union of line bundles: $H=L_{1} \oplus L_{2}$. Similarly as in the classical situation (cf. [3]) the splitting can be viewed as the field of null cones for a Lorentzian metric on $H$. Of course all such metrics are conformally equivalent. In particular, it is seen that all solutions to (6.1) are determined by the trajectories of the null field $\frac{\partial}{\partial x}+p \frac{\partial}{\partial u}+Q(x, u, p) \frac{\partial}{\partial p}$ spanning $L_{1}$.

Proposition 6.2. Fix an equation (6.1) and let $(H, g)$ be a sub-Lorentzian metric belonging to the conformal class of sub-Lorentzian metrics induced by this equation. Then any isometry of $(H, g)$ which is isotopic to the identity is in fact a point symmetry of the considered equation. 
Proof. Let $F: J^{1} \longrightarrow J^{1}$ be an isometry of $(H, g)$ as in the hypothesis of the proposition. Then, obviously, $d F(H) \subset H$ and $d F\left(L_{i}\right) \subset L_{i}, i=1,2$. Using the above notation this last remark is equivalent to the equations $F^{*} \omega^{1}=a_{1} \omega^{1}, F^{*} \omega^{2}=a_{2} \omega^{1}+a_{3} \omega^{2}, F^{*} \omega^{3}=a_{4} \omega^{1}+a_{5} \omega^{3}$ for some smooth functions $a_{i}, i=1, \ldots, 5$. If (locally) we set $F=\left(F_{1}, F_{2}, F_{3}\right)$, then the first and the third equation shows that $F_{1}$ and $F_{2}$ do not depend on $p$, which means that $F$ is the first jet prolongation of a diffeomorphism of the $(x, u)$-space. This last statement is equivalent to saying that $F$ is a point symmetry of (6.1).

Let $U$ be a neighborhood of 0 in $\mathbb{R}^{3}$ and consider any sub-Lorentzian structure $(U, H, g)$ belonging to the conformal class of equation (6.3).

Corollary 6.3. The algebra of infinitesimal isometries of $(U, H, g)$ is trivial.

Proof. Indeed, suppose that $X$ is an infinitesimal isometry with flow $\psi^{t}$. Since $\psi^{t}$ is an isometry of $(U, H, g)$ isotopic to the identity, it follows from Proposition 6.2 that $\psi^{t}$ is a point symmetry of (6.3) and therefore $\psi^{t}=\mathrm{id}$. Consequently we must have $X=0$.

We remark that the construction of sub-Lorentzian structure from an ODE as above is a particular example of a more general theory relating ODEs and what is sometimes called a para-cr structure. On this subject we refer the reader to [18].

\section{Appendix}

In this appendix we would like to draw the reader's attention to some possible applications of the invariants to non-contact cases. Consider the simplest such case, namely the Martinet case. Martinet sub-Lorentzian structures (of Hamiltonian type) were studied in [13]. Let $(M, H, g$ ) be a sub-Lorentzian manifold where $(H, g)$ is a Martinet sub-Lorentzian structure (or a metric). That is, there exists a hypersurface $S$, the so-called Martinet surface, with the following properties:

1) $H$ is a contact structure on $M \backslash S$,

2) $\operatorname{dim}\left(H_{q} \cap T_{q} S\right)=1$ for every $q \in M$,

3) the field of directions $L: S \ni q \longrightarrow L_{q}=H_{q} \cap T_{q} S$ is timelike.

It is a standard fact that trajectories of $L$ are abnormal curves for the distribution $H$. Obviously our construction of the invariants can be carried out on the contact sub-Lorentzian manifold $\left(M \backslash S, H_{\mid M \backslash S}, g_{\mid M \backslash S}\right)$. In this way we can produce necessary conditions for two Martinet sub-Lorentzian structures to be $t s$-isometric. More precisely, let $\left(M_{i}, H_{i}, g_{i}\right)$ be Martinet sub-Lorentzian manifolds such that $\left(H_{i}, g_{i}\right)$ are $t s$-oriented Martinet sub-Lorentzian metrics for $i=1,2$. Suppose that $\varphi:\left(M_{1}, H_{1}, g_{1}\right) \longrightarrow\left(M_{2}, H_{2}, g_{2}\right)$ is a $t s$-isometry, then since abnormal curves are preserved by diffeomorphisms, $\varphi\left(S_{1}\right)=S_{2}$, where $S_{i}$ is the Martinet surface for $H_{i}$, $i=1,2$. It follows that $\varphi$ induces a $t s$-isometry $\tilde{\varphi}=\varphi_{\mid M_{1} \backslash S_{1}}:\left(M_{1} \backslash S_{1}, H_{1 \mid M_{1} \backslash S_{1}}, g_{1 \mid M_{1} \backslash S_{1}}\right) \longrightarrow$ $\left(M_{2} \backslash S_{2}, H_{2 \mid M_{2} \backslash S_{2}}, g_{2 \mid M_{2} \backslash S_{2}}\right)$. Therefore, using results from Section 4 we arrive at

$$
\chi_{1}=\tilde{\varphi}^{*} \chi_{2}, \quad \kappa_{1}=\tilde{\varphi}^{*} \kappa_{2}, \quad \text { and } \quad \tilde{h}_{1}=\tilde{\varphi}^{*} \tilde{h}_{2},
$$

where $\chi_{i}, \kappa_{i}, \tilde{h}_{i}$ are the corresponding invariants for $\left(M_{i} \backslash S_{i}, H_{i \mid M_{i} \backslash S_{i}}, g_{i \mid M_{i} \backslash S_{i}}\right), i=1,2$.

As one might expect, the invariants become singular when one approaches the Martinet surface. Indeed, let us look at the following example. 
Example 7.1. Consider the simplest Martinet sub-Lorentzian structure, namely the flat one (cf. [13]). This structure is defined on $\mathbb{R}^{3}$ by the orthonormal frame

$$
X_{1}=\frac{\partial}{\partial x}+\frac{1}{2} y^{2} \frac{\partial}{\partial z}, \quad X_{2}=\frac{\partial}{\partial y}-\frac{1}{2} x y \frac{\partial}{\partial z},
$$

where we assume $X_{1}$ (resp. $X_{2}$ ) to be a time (resp. space) orientation. The Martinet surface in this case is $S=\{y=0\}$, and we can write $H=\operatorname{Span}\left\{X_{1}, X_{2}\right\}=\operatorname{ker} \omega$ for $\omega$ defined as $\omega=\frac{2}{3} \frac{1}{y} d z-\frac{1}{3} y d x+\frac{1}{3} x d y$. Clearly, $d \omega\left(X_{1}, X_{2}\right)=1$, and as usual we define the Reeb field $X_{0}$ on $\mathbb{R}^{3} \backslash S$ with equations $d \omega\left(X_{0}, \cdot\right)=0, \omega\left(X_{0}\right)=1$. Direct computation yields

$$
X_{0}=-\frac{1}{y} \frac{\partial}{\partial x}+y \frac{\partial}{\partial z} .
$$

Moreover

$$
\left[X_{2}, X_{1}\right]=\frac{1}{y} X_{1}+X_{0}, \quad\left[X_{1}, X_{0}\right]=0, \quad\left[X_{2}, X_{0}\right]=\frac{1}{y^{2}} X_{1},
$$

from which we finally obtain

$$
\tilde{h}=\left(\begin{array}{cc}
0 & \frac{1}{2} \frac{1}{y^{2}} \\
-\frac{1}{2} \frac{1}{y^{2}} & 0
\end{array}\right), \quad \chi=\frac{1}{4} \frac{1}{y^{4}}, \quad \text { and } \quad \kappa=-\frac{5}{2} \frac{1}{y^{2}} .
$$

\section{Acknowledgements}

We would like to thank the referees for their thoughtful comments and careful reading of the paper.

\section{References}

[1] Agrachev A.A., Barilari D., Sub-Riemannian structures on 3D Lie groups, J. Dyn. Control Syst. 18 (2012), 21-44, arXiv:1007.4970.

[2] Agrachev A.A., El Alaoui El-H.Ch., Gauthier J.-P., Sub-Riemannian metrics on $\mathbb{R}^{3}$, in Geometric Control and Non-Holonomic Mechanics (Mexico City, 1996), CMS Conf. Proc., Vol. 25, Amer. Math. Soc., Providence, RI, 1998, 29-78.

[3] Beem J.K., Ehrlich P.E., Easley K.L., Global Lorentzian geometry, Monographs and Textbooks in Pure and Applied Mathematics, Vol. 202, 2nd ed., Marcel Dekker, Inc., New York, 1996.

[4] Berestovskii V.N., Gichev V.M., Metrized semigroups, J. Math. Sci. 19 (2004), 10-29.

[5] Boarotto F., Conformal equivalence of sub-Riemannian 3D contact structures on Lie groups, arXiv:1412.2358.

[6] Čap A., Automorphism groups of parabolic geometries, Rend. Circ. Mat. Palermo (2005), suppl., $233-239$.

[7] El Alaoui El-H.Ch., Gauthier J.-P., Kupka I., Small sub-Riemannian balls in $\mathbb{R}^{3}$, J. Dyn. Control Syst. 2 (1996), 359-421.

[8] Falbel E., Gorodski C., Sub-Riemannian homogeneous spaces in dimensions 3 and 4, Geom. Dedicata 62 (1996), 227-252.

[9] Grochowski M., Normal forms of germs of contact sub-Lorentzian structures on $\mathbb{R}^{3}$. Differentiability of the sub-Lorentzian distance function, J. Dynam. Control Systems 9 (2003), 531-547.

[10] Grochowski M., On the Heisenberg sub-Lorentzian metric on $\mathbb{R}^{3}$, in Geometric Singularity Theory, Banach Center Publ., Vol. 65, Polish Acad. Sci., Warsaw, 2004, 57-65.

[11] Grochowski M., Reachable sets for the Heisenberg sub-Lorentzian structure on $\mathbb{R}^{3}$. An estimate for the distance function, J. Dyn. Control Syst. 12 (2006), 145-160.

[12] Grochowski M., Properties of reachable sets in the sub-Lorentzian geometry, J. Geom. Phys. 59 (2009), 885-900. 
[13] Grochowski M., Normal forms and reachable sets for analytic Martinet sub-Lorentzian structures of Hamiltonian type, J. Dyn. Control Syst. 17 (2011), 49-75.

[14] Grochowski M., Reachable sets for contact sub-Lorentzian structures on $\mathbb{R}^{3}$. Application to control affine systems on $\mathbb{R}^{3}$ with a scalar input, J. Math. Sci. 177 (2011), 383-394.

[15] Grochowski M., Remarks on global sub-Lorentzian geometry, Anal. Math. Phys. 3 (2013), 295-309.

[16] Grochowski M., Medvedev A., Warhurst B., Classification of 3-dimensional contact left-invariant subLorentzian structures, in preparation.

[17] Grong E., Vasil'ev A., Sub-Riemannian and sub-Lorentzian geometry on SU $(1,1)$ and on its universal cover, J. Geom. Mech. 3 (2011), 225-260, arXiv:0910.0945.

[18] Hill C.D., Nurowski P., Differential equations and para-CR structures, Boll. Unione Mat. Ital. 3 (2010), 25-91, arXiv:0909.2458.

[19] Huang T., Yang X., Geodesics in the Heisenberg group $H^{n}$ with a Lorentzian metric, J. Dyn. Control Syst. 18 (2012), 479-498.

[20] Korolko A., Markina I., Nonholonomic Lorentzian geometry on some H-type groups, J. Geom. Anal. 19 (2009), 864-889, arXiv:0809.4450.

[21] Korolko A., Markina I., Geodesics on $\mathbb{H}$-type quaternion groups with sub-Lorentzian metric and their physical interpretation, Complex Anal. Oper. Theory 4 (2010), 589-618, arXiv:1004.1508.

[22] Kruglikov B., The D., The gap phenomenon in parabolic geometries, arXiv:1303.1307.

[23] Liu W., Sussman H.J., Shortest paths for sub-Riemannian metrics on rank-two distributions, Mem. Amer. Math. Soc. 118 (1995), x+104 pages.

[24] Olver P.J., Equivalence, invariants, and symmetry, Cambridge University Press, Cambridge, 1995.

[25] Tanaka N., On differential systems, graded Lie algebras and pseudogroups, J. Math. Kyoto Univ. 10 (1970), 1-82. 